\title{
High oxalate levels-A potential biomarker for intense exercise
}

Running title: Biomarker for intense exercise

Sirui Yan ${ }^{1}$, Kaiqiao Yang ${ }^{1}$, Man Tang ${ }^{1}$, Yuan Wang ${ }^{1}$, Xia Huang ${ }^{1}$, Jess Lan Ouyang ${ }^{2}$, Min $\mathrm{Fu}^{3}$, Yue $\mathrm{Du}^{1}$, Qiuyun $\mathrm{Liu}^{1, *}$

${ }^{1}$ School of Life Sciences, Sun Yat-sen University, Guangzhou, China.

${ }^{2}$ Exxonmobil Chemical

${ }^{3}$ Deyang Health Committee, Deyang, Sichuan, China

\section{*Correspondence}

Qiuyun Liu, School of Life Sciences, Sun Yat-sen University, Guangzhou 510275, China

Tel: [+86 (020) 84110296]

E-mail: 1sslqy@mail.sysu.edu.cn 


\begin{abstract}
Physical exercises and labor have been confirmed in epidemiological studies that they are beneficial to human health, significantly reducing risks for cardiovascular diseases, cancer, Alzheimer's disease, etc. Despite the paucity of athletic sudden death, sporadic cases were reported each year in long distance running. Energy metabolism produces protons and organic acids in the limbs, and thus reduces stresses in internal organs. Calcium oxalate was proposed as the causative molecule of heart disease. Intensive exercise or labor can build up dangerous levels of oxalate from the pathways of energy metabolism, posing threat to few susceptible individuals. Pre-exercise or post-exercise oxalate or calcium levels, or constipation can be monitored to predict risks to cardiac arrest or athletic sudden death.
\end{abstract}

\title{
KEYWORDS:
}

Calcium oxalate, Energy metabolism, Sudden death, Intensive exercise, Warning sign 


\section{INTRODUCTION}

Physical activities tend to reduce risks for cancer and other diseases. ${ }^{1}$ According to epidemiological studies, exercises can reduce risks for cardiovascular disorders by $33 \%,{ }^{2}$ and decrease cancer risks by $10-42 \%,{ }^{3}$ Alzheimer's disease by $45 \% .{ }^{4}$ Habitual exercise becomes a lifestyle for many individuals, ranging from walking, running to cycling, etc. Exercises reduce mortality rate, and extend human lifespan. Yet, fatality among participants in marathon or long distance running events is occasionally documented. ${ }^{5}$ The cardiac arrest frequency was substantially higher during marathons (1.01 per 100,000) than during half-marathons (0.27) and among men (0.90) than among women $(0.16) .{ }^{5}$ Other epidemiological studies showed a similar trend. ${ }^{6-10}$

Physical education is compulsory in the curriculum in schools across China in order to promote student health. Yet, tragedies occur in middle-distance running including 400, 800, 1500 meter runs in middle schools and universities occasionally, demanding protective and practical measures in physical education classes for the few students at risk.

Is it possible to make the most of physical exercise while avoiding life-threatening risks? Bowel movement frequency was reported to be inversely related with cardiovascular mortality. ${ }^{11}$ Constipation is a high risk factor for both heart disease and Alzheimer's disease. ${ }^{12-13}$ Running, labor or other exercises build up lactic acid, oxalate, calcium oxalate, as well as other organic acids via the pathways of energy metabolism such as the shunt of the Krebs cycle. ${ }^{14}$ Males boast more potent energy metabolism than females on average, which is suggested by athletic performance. Previous reports indicate that people who drink modest amount of wine have lower rates of heart disease. ${ }^{15-18}$ Similar in structure to oxalate, alcohol and acetic acid are advantageous to some diseases and can extend lifespan, perhaps via the inhibition of oxalate generation. ${ }^{14,19-22}$ 
The kidneys perform many vital functions: sustaining overall fluid balance; modulating and filtering minerals from blood; filtering waste substances from food; producing hormones that help generate erythrocytes, enhance bone health, and adjust blood pressure. Renal calculi result from the buildup of calcium, oxalate, uric acid, cysteine, etc. in the kidneys. Renal stones are mostly calcium oxalate stones and sometimes lead to kidney failure and shorter lifespan. ${ }^{23}$ The ingestion of vinegar or $5 \%$ acetate increased citrate and reduced calcium in urinary excretion, and suppressed renal

formation of calcium oxalate crystals in an animal model. ${ }^{24}$ The consumption of fermented vinegar was inversely associated with kidney stone formation. ${ }^{25}$ Calcium oxalate crystals were detected within 5 hours post-death in the thyroids in $85.2 \%$ of the patients aged 70 and older. ${ }^{26}$

\section{CURRENT VIEWS ON THE MECHANISM OF THE HEALTH BENEFITS CONFERRED BY EXERCISES}

No singular pathway is involved in exercise mediated human adaptation. Various biochemical pathways as well as genetic and epigenetic regulations have been proposed to account for the benefits of physical exercises. However, the underlying mechanism is not fully clear. Factors such as mode, intensity, or duration of exercises need to be considered to improve specific metabolic or biochemical dysfunction of individuals. Variations of protein levels and mRNA levels have been observed after exercises. ${ }^{27} \mathrm{~A}$ DNA hypomethylation status was observed after exercise at specific CpG sites, ${ }^{28-29}$ and hypomethylation is associated with lower proton affinity and cation affinity, which may result in lower proton stress and slower rate on the formation of insoluble calcium salts (http://science.sciencemag.org/content/354/6314/843.6/tab-e-letters). DNA hypomethylation is also linked to up-regulations or down-regulations of gene expressions. ${ }^{30}$ 
The beneficial effects of exercises can be generated from the following: thickening of the joint capsule and ligaments; strengthening of body flexibility; thickening of muscle fibers and muscles; enhancing heart blood pumping; increasing cardiac output. ${ }^{31-32}$

\section{ADVANTAGES OF EXERCISES AND BIOCHEMICAL}

\section{PERSPECTIVE}

Moderate physical activities are advantageous to numerous diseases including heart disease and cancer. ${ }^{1}$ The Krebs cycle generates protons and organic acids, and the protons are harnessed for the production of ATP in cells. During labor or exercise, the limbs utilize protons, and the proton pool in internal organs is reduced, which lowers cancer incidences since strong acids were proposed to be a major cause of carcinogenesis. Calcium supplement neutralizes $\mathrm{HCl}$ and can decrease cancer risk. ${ }^{33}$ Moderate exercise consumes energy source and reduces the generation of oxalate via energy metabolism in internal organs, thus demonstrating favorable effects to cardiovascular system. Excessive reading or online surfing renders some individuals near sightedness, perhaps through the buildup of rigid calcium oxalate. Exercise can also improve vision ${ }^{34}$, likely via the diversion of organic acids and protons to the limbs.

\section{STRATEGIES TO REDUCE ATHLETIC SUDDEN DEATH}

People experiencing regular constipation may have higher level of the insoluble and rigid calcium oxalate, and consequently have greater likelihood of developing heart problems during intense physical activities. ${ }^{33,35}$ Calcium oxalate was proposed as the potential cause of death. ${ }^{19,26,33,35-36}$ Therefore, it can be adopted as a signatory molecule for foretelling post-running or post-exercise physical conditions. Pre-exercise or postexercise testing of dangerous oxalate or calcium levels can be valuable to susceptible individuals, and constipation could serve as an early warning sign for indications of potential risks for intensive labor or exhaustive exercises to reduce human casualty. Other established cardiovascular risk factors such as obesity, diabetes mellitus, hypertension, hyperlipidemia are also concerns related to sudden death. ${ }^{37}$ 


\section{NUTRITIONAL TIPS BEFORE EXERCISE OR LABOR}

Plant-based diet reduces the intake of essential amino acids such as valine, and valineand glycine-rich proteins were postulated to possess calcium-binding properties and to be the causative factors of heart disease. ${ }^{33,38}$ Weak organic acid-rich diet such as fruits, vegetables and yogurt solubilizes the insoluble salts, reduces stresses, and is beneficial to individuals. ${ }^{33,39}$

The benefits of high-fiber diet have been widely documented. ${ }^{40}$ Dietary fibers are rich in hydroxyl groups and aldehyde groups with potent secondary bonding to cations on the oxygen atoms. The affinity to protons is prone to generate strong acids such as $\mathrm{HCl}$. The bonding to non-proton cations in dietary fibers may produce insoluble and rigid salts such as calcium oxalate. The key to dietary fibers' advantages is that they are macromolecules in various forms, which do not penetrate into the inside of cells and hence carry away the disease-causing cations and insoluble salts via the digestive tract. ${ }^{41}$ This sets dietary fibers apart from mono-, di-, or tri-saccharides in the health benefits to humans after consumption. The favorable microbiome profile that dietary fibers generate is also advantageous, as acetate and butyrate produced from the gut microbiota counteract strong acids and solubilize insoluble and rigid salts in some circumstances. $^{42}$

\section{CONCLUSION}

Numerous biochemical pathways, genetic and epigenetic regulations are involved in exercise-mediated adaptation, suggesting a broad molecular response of the human body. Yet, exhaustive physical exercises may accumulate dangerous levels of oxalate from the pathways of energy metabolism in few susceptible individuals, potentially triggering cardiac arrest or athletic sudden death. Oxalate levels can be monitored to confirm association with athletic sudden death. The presence of insoluble and rigid salts such as calcium oxalate sometimes is manifested in the gastrointestinal system in the 
form of constipation.

This hypothesis addresses an important point in athletic sudden death but the relative paucity of different of sudden death reports during physical labor/exercise should be considered. Given the extensive benefits of physical exercise to human health, this hypothesis should be regarded as a supplement to current views. The use of oxalate as a biomarker for cardiac arrest or athletic sudden death needs to be substantiated by epidemiological and biochemical studies.

\section{ACKNOWLEDGMENT}

This work was supported by grants from the Science and Technology Transformation Program of Sun Yat-sen University of China (33000-18843234) and Guangzhou Science and Technology Program (201804010328) to Q.L; Guangdong Natural Science Foundation (2018A030313294) to X.H. We are grateful to helpful discussions with Jiaming Zhang, Weiguo Cao and Yuchuan Wang, and editing by Yan Shi.

\section{CONFLICT OF INTEREST}

The author declares no conflicts of interest to disclose.

\section{REFERENCES}

1. Zhou Y, Xing M, Li X, et al. Physical exercises and heart health. Eur J Prev Cardiol. $2018 ; 25: 639$.

2. Morris JN, Chave SP, Adam C, et al. Vigorous exercise in leisure-time and the incidence of coronary heart-disease. Lancet. 1973;1: 333-339.

3. Moore SC, Lee IM, Weiderpass E, et al. Association of leisure-time physical activity with risk of 26 types of cancer in 1.44 million adults. JAMA Intern Med. 2016;176(6):816-825.

4. Hamer M, Chida Y. Physical activity and risk of neurodegenerative disease: a systematic review of prospective evidence. Psychol Med. 2009;39(1):3-11. 
5. Kim JH, Malhotra R, Chiampas G, et al. Cardiac arrest during long-distance running races. $N$ Engl J Med. 2012;366(2):130-40.

6. Jiménez-Pavón D, Lavie CJ, Blair SN. The role of cardiorespiratory fitness on the risk of sudden cardiac death at the population level: A systematic review and metaanalysis of the available evidence. Prog Cardiovasc Dis. 2019;62(3): 279-287.

7. Schwellnus MP. Premarathon evaluations: is there a role for runner prerace medical screening and education to reduce the risk of medical complications? Curr Sports Med Rep. 2017;16(3):129-136.

8. Harmon KG, Zigman M, Drezner JA. The effectiveness of screening history, physical exam, and ECG to detect potentially lethal cardiac disorders in athletes: a systematic review/meta-analysis. J Electrocardiol. 2015;48(3):329-38.

9. Sealy DP, Pekarek L, Russ D, Sealy C, Goforth G. Vital signs and demographics in the preparticipation sports exam: do they help us find the elusive athlete at risk for sudden cardiac death? Curr Sports Med Rep. 2010;9(6):338-41.

10. Link MS, Estes NA. Athletes and arrhythmias. J Cardiovasc Electrophysiol 2010; 21(10):1184-9.

11. Vermorken AJ, Andrès E, Cui Y. Bowel movement frequency, oxidative stress and disease prevention. Mol Clin Oncol. 2016;5:339-342.

12. Salmoirago-Blotcher E, Crawford S, Jackson E, et al. Constipation and risk of cardiovascular disease among postmenopausal women. Am J Med. 2011;124: 714723.

13. Coggrave M, Norton C, Cody J D. Management of faecal incontinence and constipation in adults with central neurological diseases. Cochrane Database Syst Rev. 2014;1:CD002115.

14. Shi Y, Wan Y, Zhou Y, et al. Why various wines reduce the risks of heart diseases. Eur J Prev Cardiol. 2017;24:1646-1647.

15. Chalons P, Amor S, Courtaut F, et al. Study of potential anti-inflammatory effects of red wine extract and resveratrol through a modulation of interleukin-1-beta in macrophages. Nutrients 2018;10(12):1856. 
16. Liberale L, Bonaventura A, Montecucco F, Dallegri F, Carbone F. Impact of red wine consumption on cardiovascular health. Curr Med Chem.2019;26(19): 35423566.

17. Sato M, Maulik N, Das DK. Cardioprotection with alcohol: role of both alcohol and polyphenolic antioxidants. Ann N Y Acad Sci. 2002; 957: 122-135.

18. Das DK, Sato M, Ray PS, et al. Cardioprotection of red wine: role of polyphenolic antioxidants. Drugs Exp Clin Res. 1999;25(2-3):115-120.

19. An S, Li X, Tang M, et al. Functional duality of ethanol on cancer. Med Hypotheses. 2019;122:124-125.

20. Chuang $\mathrm{MH}$, Chiou $\mathrm{SH}$, Huang $\mathrm{CH}$, et al. The lifespan-promoting effect of acetic acid and Reishi polysaccharide. Bioorg Med Chem. 2009;17:7831-7840.

21. Paganini-Hill A, Kawas CH, Corrada MM. Type of alcohol consumed, changes in intake over time and mortality: the Leisure World Cohort Study. Age Ageing. 2007;36:203-209.

22. Ali Z, Wang Z, Amir RM, et al. Potential uses of vinegar as a medicine and related in vivo mechanisms. Int J Vitam Nutr Res. 2016;86:127-151.

23. Hall JA, Yerramilli M, Obare E, et al. Serum concentrations of symmetric dimethylarginine and creatinine in cats with kidney stones. PLoS One. 2017;12: e0174854.

24. Zhu W, Liu Y, Lan Y, et al. Dietary vinegar prevents kidney stone recurrence via epigenetic regulations. EBioMedicine. 2019;45:231-250.

25. Zeng G, Mai Z, Xia S, et al. Prevalence of kidney stones in China: an ultrasonography based cross-sectional study. BJU Int. 2017;120(1):109-116.

26. Katoh R, Suzuki K, Hemmi A, et al. Nature and significance of calcium oxalate crystals in normal human thyroid gland. A clinicopathological and immunohistochemical study. Virchows Arch A Pathol Anat Histopathol. 1993; 422: $301-306$

27. Maejima H, Ninuma S, Okuda A, Inoue T, Hayashi M. Exercise and low-level GABAA receptor inhibition modulate locomotor activity and the expression of BDNF accompanied by changes in epigenetic regulation in the hippocampus. 
Neurosci Letters. 2018;685:18-23.

28. da Silva IRV, de Araujo CLP, Dorneles GP, et al. Exercise-modulated epigenetic markers and inflammatory response in COPD individuals: a pilot study. Respir Physiol Neurobiol. 2017;242:89-95.

29. Sølvsten CAE, de Paoli F, Christensen JH, Nielsen AL. Voluntary physical exercise induces expression and epigenetic remodeling of VegfA in the rat hippocampus. Mol Neurobiol. 2018;55(1):567-582.

30. Wilson AS, Power BE, Molloy PL. DNA hypomethylation and human diseases. Biochim Biophys Acta. 2007; 1775(1):138-162.

31. Denham J. Exercise and epigenetic inheritance of disease risk. Acta Physiol (Oxf). $2018 ; 222(1)$.

32. Egan B, Zierath JR. Exercise metabolism and the molecular regulation of skeletal muscle adaptation. Cell Metab. 2013;17:162-184.

33. Wan Y, Zhang J, Li X, et al. Cellular states and secondary chemical bonding: a biochemical view of major human diseases. Biochem Insights. 2019;12: 1178626419877846.

34. Pardue MT, Chrenek MA, Schmidt RH, Nickerson JM, Boatright JH. Potential role of exercise in retinal health. Prog Mol Biol Transi Sci 2015;134:491-502.

35. Wan Y, Zou J, Shi Y, et al. How to avoid cardiac sudden death. Eur J Prev Cardiol. $2017 ; 24: 1790$.

36. An S, Li X, Tang M, et al. The role of acetate in the antagonism of oxalate - A potential causative molecule for heart disease and cancer death. Nat Prod Commun. 2020;15(3):1-4

37. Jayaraman R, Reinier K, Nair S, et al. Risk factors of sudden cardiac death in the young: multiple-year community-wide assessment. Circulation.2018;137: 15611570 .

38. Qi J, Zhang X, Chen Z, et al. Transgenic proteins rich in valine or glycine are concerns for heart disease patients. Eur J Prev Cardiol. 2018;25: 883-884.

39. Zhou Y, Shi Y, Wan Y, et al. Why the Mediterranean diet lowers the risk of heart disease. Eur J Prev Cardiol. 2017;24:1788-1789. 
40. Rubin R. High-Fiber Diet Might Protect Against Range of Conditions. JAMA. 2019;321:1653-1655.

41. Tang M, Zhou Y, Li Y, et al. Hydrogen donors and acceptors and basic amino acids jointly contribute to carcinogenesis. Med Hypotheses. 2017;98:42-44.

42. Hu X, Xing M, An S, et al. The gut microbiota and heart disease prevention. Eur J Prev Cardiol. 2019;26:109. 